\title{
A bipartite graph with non-unimodal independent set sequence
}

\author{
Arnab Bhattacharyya \\ DIMACS \& Dept. of Computer Science \\ Rutgers University \\ New Jersey, U.S.A. \\ arnabb@dimacs.rutgers .edu
}

\author{
Jeff Kahn \\ Dept. of Mathematics \\ Rutgers University \\ New Jersey, U.S.A. \\ jkahn@math.rutgers.edu
}

Submitted: Apr 7, 2013; Accepted: Oct 16, 2013; Published: Oct 28, 2013

Mathematics Subject Classifications: 05C69, 05C31

\begin{abstract}
We show that the independent set sequence of a bipartite graph need not be unimodal.
\end{abstract}

\section{Introduction}

For a graph $G=(V, E)$ and an integer $t \geqslant 0$, let $i_{t}(G)$ denote the number of independent sets of size $t$ in $G$. (Recall that an independent set is a set of vertices spanning no edges.) The independent set sequence of $G$ is the sequence $i(G)=\left(i_{t}(G)\right)_{t=0}^{\alpha(G)}$, where $\alpha(G)$ is the size of a largest independent set in $G$.

It was conjectured by Levit and Mandrescu [LM06] that for any bipartite graph $G$, $i(G)$ is unimodal; that is, that there is a $k$ for which

$$
i_{0}(G) \leqslant i_{1}(G) \leqslant \cdots \leqslant i_{k}(G) \geqslant i_{k+1}(G) \geqslant \cdots \geqslant i_{\alpha(G)}(G) .
$$

Evidence in favor of this was given by Levit and Mandrescu [LM06] and by Galvin (in [Gal12], which got us interested in the problem, and in [Gal11]).

In this note, we disprove the conjecture:

Theorem 1. There are bipartite graphs $G$ for which $i(G)$ is not unimodal.

Still open and very interesting is the possibility, first suggested by Alavi, Malde, Schwenk and Erdős [AMSE87], that trees and forests have unimodal independent set sequences. See also [Sta89] for a general survey of unimodality and the stronger notion of log-concavity. 


\section{Counterexample}

Given positive integers $a$ and $b>a$, let $G=G(a, b)=(V, E)$ with: $V=V_{1} \cup V_{2} \cup V_{3}$, where $V_{1}, V_{2}, V_{3}$ are disjoint; $\left|V_{1}\right|=b-a$ and $\left|V_{2}\right|=\left|V_{3}\right|=a$; and $E$ consists of a complete bipartite graph between $V_{1}$ and $V_{2}$ and a perfect matching between $V_{2}$ and $V_{3}$.

Lemma 2. For every $t \geqslant 0, i_{t}(G)=\left(2^{t}-1\right)\left(\begin{array}{l}a \\ t\end{array}\right)+\left(\begin{array}{l}b \\ t\end{array}\right)$.

Proof. Each independent set in $G$ is a subset of either $V_{1} \cup V_{3}$ or $V_{2} \cup V_{3}$. Among independent sets of size $t$, the number of the first type is $\left(\begin{array}{l}b \\ t\end{array}\right)$, the number of the second type is $2^{t}\left(\begin{array}{l}a \\ t\end{array}\right)$, and the number that are of both types (that is, that are subsets of $\left.V_{3}\right)$ is $\left(\begin{array}{l}a \\ t\end{array}\right)$.

We now assert that $i(G)$ is not unimodal if $a$ is large and (say) $b=\left\lfloor a \log _{2} 3\right\rfloor$. In this case, the expressions $\left(\begin{array}{l}b \\ t\end{array}\right)$ and $2^{t}\left(\begin{array}{l}a \\ t\end{array}\right)$ are maximized at $t_{1}=b / 2$ and $t_{2}=2 a / 3+O(1)$ respectively (the overlap $\left(\begin{array}{l}a \\ t\end{array}\right)$ is negligible), with each maximum on the order of $3^{a} / \sqrt{a}$. On the other hand, each expression is $o\left(3^{a} / \sqrt{a}\right)$ if $t$ is at least $\omega(\sqrt{a})$ from the maximizing value. In particular, $i_{t}(G)$ is much smaller for $t=\left(t_{1}+t_{2}\right) / 2$ than for $t \in\left\{t_{1}, t_{2}\right\}$, so $i(G)$ is not unimodal.

For a concrete example, we may take $a=95$ and $b=151$, for which explicit calculation gives

$$
\begin{aligned}
& i_{70}(G)=189874416016052359845764115146202643360315069 \\
& i_{71}(G)=187958904435447560369145399619337946363249075 \\
& i_{72}(G)=188299580501161488791208803278091384597416875
\end{aligned}
$$

In fact, we find that 95 is the minimum value of $a$ for which the above construction produces a counterexample.

\section{Remarks}

The construction above can be generalized to show that (for bipartite $G$ ) $i(G)$ can have arbitrarily many local maxima. Given (positive) integers $k$ and $a, a_{1}, \ldots, a_{k}$, let $G=$ $G\left(a, a_{1}, \ldots, a_{k}\right)=(A \cup B, E)$ be the bipartite graph where: $A=\cup_{i=0}^{k} A_{i}$ and $B=\cup_{j=1}^{k} B_{j}$, with all $A_{i}$ 's and $B_{j}$ 's disjoint; $\left|A_{0}\right|=\left|B_{1}\right|=\left|B_{2}\right|=\cdots=\left|B_{k}\right|=a$ and $\left|A_{i}\right|=a_{i}$ for $i>0$; and $E$ consists of a perfect matching between $A_{0}$ and $B_{j}$ for each $j>0$, together with a complete bipartite graph between $A_{i}$ and $B_{j}$ for all $(i, j)$ with $j \leqslant i$. Then for $a, a_{1}, \ldots, a_{k}$ large with all the $k+1$ expressions $2^{a_{1}+\cdots+a_{i}}\left(1+2^{k-i}\right)^{a}$ roughly equal, an analysis similar to the one above shows that $i(G)$ has $k+1$ local maxima.

\section{References}

[AMSE87] Y. Alavi, P.J. Malde, A.J. Schwenk, and P. Erdős. The vertex independence sequence of a graph is not constrained. Congressus Numerantium, 58:15-23, 1987. 
[Gal11] D. Galvin. Two problems on independent sets in graphs. Discrete Mathematics, 311(20):2105-2112, 2011.

[Gal12] D. Galvin. The independent set sequence of regular bipartite graphs. Discrete Mathematics, 312:2881-2892, 2012.

[LM06] V.E. Levit and E. Mandrescu. Partial unimodality for independence polynomials of König-Egerváry graphs. Congressus Numerantium, 179:109-119, 2006.

[Sta89] R.P. Stanley. Log-concave and unimodal sequences in algebra, combinatorics, and geometry. Annals of the New York Academy of Sciences, 576:500-535, 1989 . 